\author{
Costas Panayotidis - Suhaiza Salleh · Jose E. Martin • \\ Peter Hirsh · John Wynn
}

\title{
Giant uterine leiomyomas: dilemmas in surgical management
}

Published online: 13 December 2005

(C) Springer-Verlag Berlin/Heidelberg 2005

\begin{abstract}
Giant fibroids are very rare in current practice, posing a management challenge. Careful consideration of the benefits and limitations of each treatment option must be considered. This article summarises current management of giant leiomyomas, with a clinical case example and recent review of the literature.
\end{abstract}

Keywords Giant uterine leiomyomas · Laparotomy · Laparoscopy $\cdot$ Uterine artery embolisation $\cdot \mathrm{GnRH}$

\section{Case study}

A 37-year-old nulliparous woman was referred to the gynaecology outpatient clinic in 1999, with a pelvic mass and pressure symptoms. She had regular periods with no history of menorrhagia or dysmenorrhoea. Clinical examination revealed a large abdominal mass, most likely of uterine origin. Transabdominal ultrasound scan (USS) confirmed the presence of a grossly enlarged uterus containing at least ten areas of mixed echogenicity consistent with fibroids. The largest of these measured $14 \times 13 \mathrm{~cm}$ and extended above the umbilicus to about $12 \mathrm{~cm}$.

Myomectomy, with its inherent risk of hysterectomy, was declined by the patient as she wished to retain her fertility. A 3-month medical treatment with gonadotrophin releasing hormone $(\mathrm{GnRH})$ analogues was tried in an attempt to reduce the size of the uterus. A repeat USS posttreatment showed virtually no change in the size of the fibroids. She was referred for a radiological opinion to consider uterine artery embolisation, but it was felt that the treatment would be unsuitable because even if a $50 \%$ reduction in the size of the fibroids was obtained, the pressure effect of the uterus would still be substantial. There were also concerns regarding severe post-embolisa-

C. Panayotidis $(\bowtie) \cdot$ S. Salleh · J. E. Martin · P. Hirsh · J. Wynn Department of Obstetrics and Gynaecology, South Manchester University Hospitals NHS Trust,

Wythenshawe, Manchester, UK

e-mail: costapan@hotmail.com tion syndrome and a higher than normal risk of complications requiring a hysterectomy. At this point, the patient declined further interventions; therefore, annual gynaecological follow-up with USS examinations was arranged. Over the following years, the fibroids increased in size, and the patient finally opted for definitive surgical treatment. A total abdominal hysterectomy with conservation of the ovaries was performed in 2005. A 3-month GnRH analogue treatment was given preoperatively. A paramedian skin incision was chosen and a myomectomy was performed before proceeding to hysterectomy. The intraoperative estimated blood loss was $600 \mathrm{ml}$. She had 2 units of blood transfused postoperatively and had an uneventful recovery period. The pathological examination of the specimen showed a grossly distorted uterine body with multiple fibroids. The entire specimen weighed $4550 \mathrm{~g}$ and measured $31 \times 26 \times 15 \mathrm{~cm}$, fetal-shaped fibroid uterus (Fig. 1). Histological examination confirmed the diagnosis of leiomyomas with no evidence of malignancy.

\section{Discussion}

Leiomyomas, or fibroids, are the commonest solid, benign pelvic tumours and it is estimated that $20-30 \%$ of all women have one or more present at post-mortem, the vast majority of these having been symptomless [1]. Despite the high incidence, it is uncommon to encounter a large fibroid as in this case, probably due to the availability of a wide variety of treatment options and early resource to treatment. Nevertheless, large fibroids can be non-troublesome as in this case, and present with minimal pressure symptoms or patients' concern regarding "abdominal enlargement" $[2,3]$.

The current, established management of uterine fibroids may involve one of the following approaches or a combination thereof: expectant management, surgical management, medical management or uterine artery embolisation. The chosen approach should be individualised depending on various factors, including age, type and severity of symptoms, suspicion of malignancy, desire for 
Fig. 1 a-d Fetal shaped giant fibroid uterus a

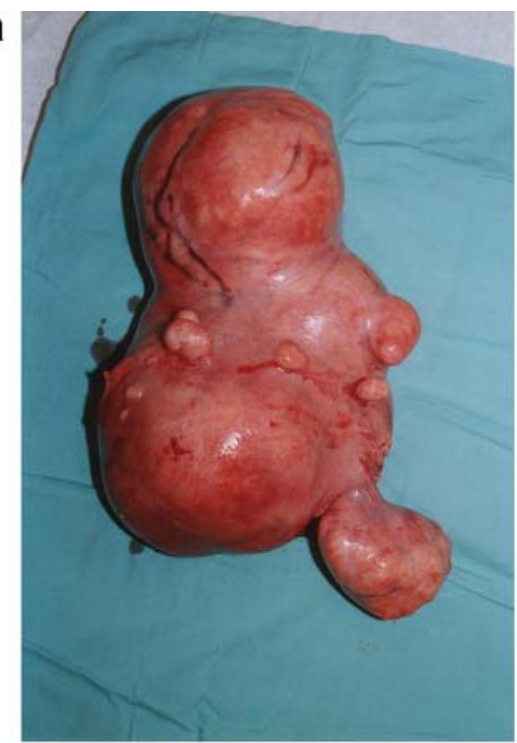

C

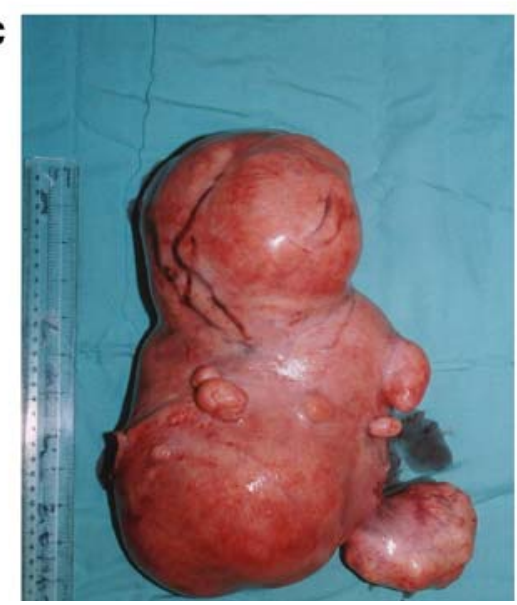

b

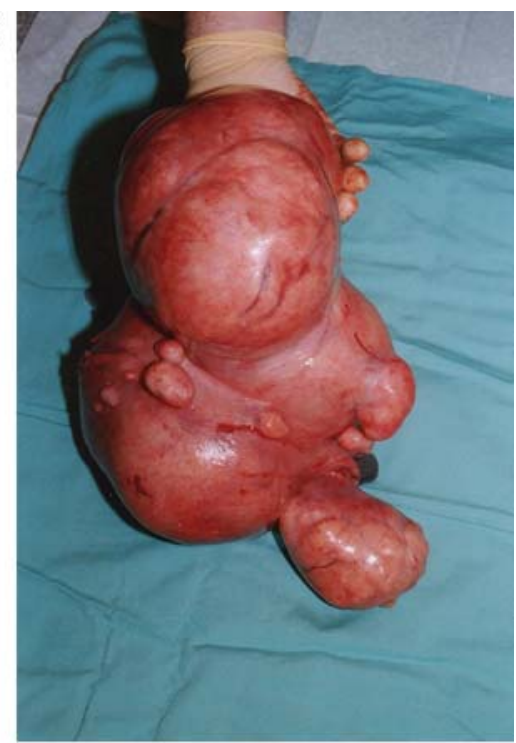

d

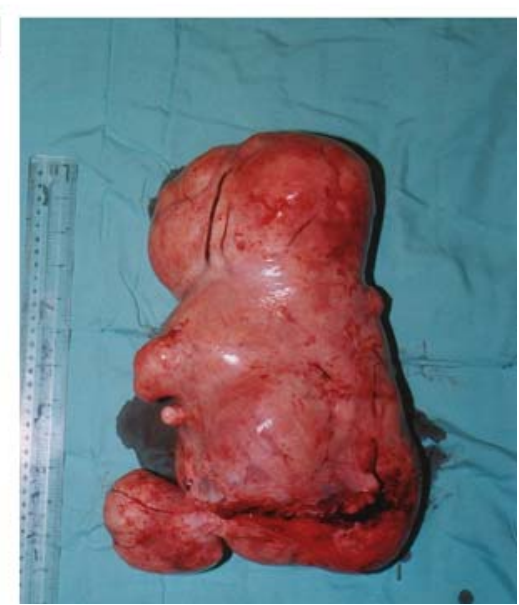

future fertility and proximity to the menopause. In our case, the patient's desire to retain fertility and the absence of significant symptoms were the main factor that influencing the management.

\section{Expectant management}

Despite the uterine size, expectant management may be the management of choice. In this case, it was chosen by the patient after failure of medical treatment, unsuitability of uterine artery embolisation and the reluctance to proceed to surgical intervention. The intervals between follow-ups should depend on individual cases. The expectant approach is clearly applicable to women approaching menopause.

\section{Surgical management}

The indications for surgical management of uterine fibroids includes abnormal bleeding which is not responding to conservative treatments, iron deficiency anaemia second- ary to chronic bleeding, pain or pressure symptoms that interfere with quality of life, high suspicion of malignancy, subfertility when there is distortion of the endometrial cavity or tubal occlusion and recurrent pregnancy loss with distortion of the endometrial cavity [4]. A laparoscopic procedure can be attempted where myomectomy and/or myolysis using a uterine morcellator, is performed. However, this approach is applicable only for smaller fibroid uteri. In addition, laparoscopically assisted vaginal hysterectomy is an option for experienced laparoscopists, with successful results. The surgical management of tumours of large calibre $(>25 \mathrm{~cm})$, as in our case, can be a challenge even when the open surgical approach is chosen, and again in these cases a laparoscopic method is not recommended. Pre-operative assessment should be meticulous, in order to avoid intra-operative and post-operative complications.

\section{Pre-operative phase}

Size, anatomical position, distortion of adjacent organs and features suggestive of malignancy can be assessed using 
various imaging techniques. In the majority of cases USS is the examination of choice. However, in our case, due to the presence of a very large tumour, only partial sonographic measurements could be made. This is when computed tomography (CT) or magnetic resonance imaging (MRI) can provide a better estimation of the pelvic topography as well as accurate measurements of the leiomyoma itself. In specific cases, CT can supplement ultrasonography, although it has been suggested that up to $5 \%$ of patients reported to have uterine fibroids by CT were subsequently found to have ovarian tumours at the time of surgery [5]. Magnetic resonance imaging provides better discrimination of the source of the pelvic mass [6]. Other pre-operative measures include correction of anaemia, optimising body mass index in obese patients and anaesthetic assessment in patients with high anaesthetic risk. The use of GnRH analogues for 3 or 4 months prior to fibroid surgery (hysterectomy or myomectomy) has been shown to reduce both uterine volume and fibroid size, correct iron deficiency anaemia, reduce intra-operative blood loss, diminish the need to perform a midline incision and increas the possibility of vaginal hysterectomy in selected cases $[7,9]$. Little is known about volume reduction for giant fibroids when $\mathrm{GnRH}$ analogues have been used. For fibroid uteri of such large size, the effect may not optimise the surgery significantly. Careful selection of patients is necessary in order to avoid unnecessary side effects from $\mathrm{GnRH}$ agonists. Concerning uterine arterial embolisation (UAE), reports have shown that selective use of UAE as an adjunct prior to hysterectomy or myomectomy may be useful in reducing the size of the leiomyomas and help limiting intraoperative blood loss $[2,8]$.

\section{Intra-operative phase}

The patient must be positioned so that superior vena cava compression is avoided and ventilation not impaired. Increased blood loss must be anticipated and adequate supply of cross matched blood must be available throughout procedure. The abdominal incision chosen must provide optimal exposure for manipulating and dissecting such large masses originating in the pelvis. In the majority of cases, a longitudinal abdominal incision is advisable. The uterus may be exteriorised or a myomectomy screw may be used for easier handling. In certain cases, other procedures, for example, enucleation of the tumour or hemi-section of the uterus, may be necessary before the hysterectomy can be performed. When UAE is used prior to the surgery, the surgeon could locate the arteries that feed the larger fibroids and place a large clamp forceps on the ipsilateral side of uterus. This application can promptly decrease the amount of bleeding during dissection. Pelvic anatomical distortion due to the presence of an abnormal uterus increases the risk of injury to adjacent structures including the bowel and the urinary tract. Bladder and/or ureteric accidents can occur when the tumour extends into their proximity. Therefore, careful dissection of the area below the broad ligament and visualisation of the ureters prior to application of surgical clamps is advised. The cervix may also be distorted by leiomyomas, resulting in difficult dissection of the utero-vesical peritoneal fold which may lead to urinary bladder injury. In certain cases, subtotal hysterectomy may be a "safer" option. The surgery may not technically differ whether we have to deal with a large unilateral or a multiple fibroid uterus. The surgical management of these cases may need a multidisciplinary approach, involving not only the gynaecologist but also the urologist, as well as gastrointestinal surgeons.

\section{Medical treatment}

The use of GnRH analogues is limited from the point of view that its beneficial effect of uterine volume reduction (50\% reduction of initial volume within 3 months of therapy), is temporary, with re-growth of fibroids within 12 weeks of stopping treatment [9]. Little evidence exists regarding their effect on uterine volume reduction, as in our case. GnRH analogue treatment should be restricted to a 3to 6-month interval as longer use is associated with osteoporosis [9]. The unpleasant hypo-estrogenic side effects also reduce the compliance in many patients.

\section{Uterine artery embolisation}

UAE is an alternative treatment in selected cases with symptomatic fibroids. Women choosing this treatment should be made aware of the side effects and risks, which include acute and chronic abdominal pain, post-embolisation syndrome, infection, ovarian dysfunction, menstrual dysfunction, hysterectomy and mortality. They also need to be counselled regarding lack of long-term data on efficacy, fecundity, pregnancy outcome and long-term patient satisfaction [10]. In the presence of large fibroids, only improvement in menstrual loss is achieved after UEA and no significant reduction in the size of the fibroids, therefore UEA is not recommended for the management of larger fibroids where there is also a significant increase in the rates of complications $[11,12]$. Therefore, if the predominant complaint is that of a mass, as in our case, alternative treatments need to be considered.

\section{Conclusion}

Giant fibroids are very rare in current practice, posing a management challenge. Careful consideration of the benefits and limitations of each treatment option (surgical, medical or a combination), should be considered. Treatment should be individualised, taking into consideration important issues like the patient's wishes to retain fertility. Surgical management may be difficult and a multidisciplinary approach may be needed. 


\section{References}

1. Whitfield CR (ed) (1996) Dewhurst's textbook of obstetrics and gynaecology for postgraduates, 5th edn. Blackwell, London, pp 739-746

2. Djelmis J, Mayer D, Majerovic M, Radanovic B, Starcevic V (2001) Giant uterine leiomyoma devascularized by embolization prior to surgical removal. Eur J Obstet Gynecol Reprod Biol 99(2):278-280

3. Evans AT III, Pratt JH (1979) A giant fibroid uterus. Obstet Gynecol 54(3):385-386

4. Wallach EE, Vlahos NF (2004) Uterine myomas: an overview of development, clinical features, and management. Obstet Gynecol 104(2):393-406

5. Tada S, Tsukioka M, Ishii C, Tanaka H, Mizunuma K (1981) Computed tomographic features of uterine myoma. J Comput Assist Tomogr 5(6):866-869

6. Scoutt LM, McCarthy SM (1990) Applications of magnetic resonance imaging to gynecology. Top Magn Reson Imaging 2 (3):37-49

7. Lethaby A, Vollenhoven B, Sowter M (2002) Efficacy of preoperative gonadotrophin hormone releasing analogues for women with uterine fibroids undergoing hysterectomy or myomectomy: a systematic review. Br J Obstet Gynaecol 109 (10):1097-1108

8. Ngeh N, Belli AM, Morgan R, Manyonda I (2004) Premyomectomy uterine artery embolisation minimises operative blood loss. Br J Obstet Gynaecol 111(10):1139-1140

9. Vercellini P, Crosignani PG, Mangioni C, Imparato E, Ferrari A, De Giorgi O (1998) Treatment with a gonadotrophin releasing hormone agonist before hysterectomy for leiomyomas: results of a multicentre, randomised controlled trial. Br J Obstet Gynaecol 105(11):1148-1154

10. Lefebvre G, Vilos G, Allaire C, Jeffrey J, Arneja J, Birch C, Fortier M, Wagner MS, Clinical Practice Gynaecology Committee, Society for Obstetricians and Gynaecologists of Canada (2003) The management of uterine leiomyomas. J Obstet Gynaecol Can 25(5):396-418; quiz 419-422

11. Prollius A, de Vries C, Loggenberg E, du Plessis A, Nel M, Wessels PH (2004) Uterine artery embolisation for symptomatic fibroids: the effect of the large uterus on outcome. Br J Obstet Gynaecol 111(3):239-242

12. McLucas B et al (1999) Fatal septicaemia after fibroid embolisation. Lancet 354:1571-1575 